\title{
Prospective Study of Fertility Preservation in Young Women With Breast Cancer in Mexico
}

\author{
Cynthia Villarreal-Garza, MD, DSc $c^{1,2}$; Fernanda Mesa-Chavez, MD ${ }^{1,2}$; Alejandra Plata de la Mora, Psy, MSc ${ }^{2,3}$; \\ Melina Miaja-Avila, Psy, $\mathrm{PhD}^{1,2}$; Marisol Garcia-Garcia, MD ${ }^{1,2}$; Alan Fonseca, MD², \\ Sylvia de la Rosa-Pacheco, MD, MSc ${ }^{4}$; Marlid Cruz-Ramos, MD, PhD ${ }^{2,3}$; Manuel Rolando García Garza, MD; \\ Alejandro Mohar, MD, DSc ${ }^{2,6,7}$; and Enrique Bargallo-Rocha, $\mathrm{MD}^{2,3}$
}

\begin{abstract}
Background: Despite the risk of treatment-related infertility, implementation of fertility-preservation (FP) strategies among young patients with breast cancer is often suboptimal in resource-constrained settings such as Mexico. The "Joven \& Fuerte: Program for Young Women With Breast Cancer" strives to enhance patient access to supportive care services, including FP measures through alliances with assisted-reproduction units and procurement of coverage of some of these strategies. This study describes patients from Joven \& Fuerte who have preserved fertility, and assesses which characteristics were associated with the likelihood of undergoing FP. Methods: Women aged $\leq 40$ years with recently diagnosed breast cancer were prospectively accrued. Sociodemographic and clinicopathologic data were collected from patient-reported and provider-recorded information at diagnosis and 1-year follow-up. Descriptive statistics, chi-square test, and simple logistic regression were used to compare patients who preserved fertility with those who did not. Results: In total, 447 patients were included, among which 53 (12\%) preserved fertility, representing $38 \%$ of the 140 women who desired future biologic children. Oocyte/embryo cryopreservation was the most frequently used method for FP (59\%), followed by temporary ovarian suppression with gonadotropin-releasing hormone agonists $(\mathrm{GnRHa})$ during chemotherapy $(26 \%)$, and use of both $\mathrm{GnRHa}$ and oocyte/embryo cryopreservation (15\%). Younger age, higher educational level, being employed, having private healthcare insurance, and having one or no children were associated with a significantly higher likelihood of preserving fertility. Conclusions: By facilitating referral and seeking funds and special discounts for underserved patients, supportive care programs for young women with breast cancer can play a crucial role on enhancing access to oncofertility services that would otherwise be prohibitive because of their high costs, particularly in resource-constrained settings. For these efforts to be successful and widely applied in the long term, sustained and extended governmental coverage of FP options for this young group is warranted.
\end{abstract}

J Natl Compr Canc Netw, doi: 10.6004/jnccn.2020.7692 Published online June 21, 2021

\footnotetext{
${ }^{1}$ Centro de Cancer de Mama, TecSalud, Tecnologico de Monterrey, San Pedro Garza Garcia, Nuevo Leon; ${ }^{2}$ Joven \& Fuerte, Programa para la Atencion e Investigacion para Pacientes Jovenes con Cancer de Mama en Mexico, Ciudad de Mexico; ${ }^{3}$ Departamento de Tumores Mamarios e Investigacion, Instituto Nacional de Cancerologia, Ciudad de Mexico; ${ }^{4}$ Tecnologico de Monterrey, Escuela de Medicina y Ciencias de la Salud, Monterrey, Nuevo Leon; ${ }^{5}$ Instituto de Ginecologia, TecSalud, Tecnologico de Monterrey, Monterrey, Nuevo Leon; and ${ }^{6}$ Unidad de Epidemiologia e Investigacion Biomedica en Cancer, Instituto Nacional de Cancerologia, and ${ }^{7}$ Instituto de Biomedicas, Universidad Nacional Autonoma de Mexico, Ciudad de Mexico, México.
}

\section{Background}

Breast cancer is the most commonly diagnosed malignancy among women of childbearing age. ${ }^{1}$ In developed countries, approximately $7 \%$ of breast cancer cases are diagnosed in young women, ${ }^{2}$ whereas in less thriving settings such as Mexico this rate reaches up to $15 \%{ }^{3}$

Among the unique age-related challenges that young women with breast cancer (YWBC) face, almost half report unmet parity at diagnosis, a rate that has been reproduced in Mexican patients. ${ }^{4,5}$ Therefore, potential treatment-related premature ovarian failure and fertility loss are particularly relevant and can cause significant psychologic distress in this group. ${ }^{6,7}$

For this reason, national and international guidelines recommend that physicians discuss the treatment-related risk of infertility with patients of reproductive age to aid them with fertility preservation (FP) decisions as early as possible. ${ }^{8-10}$ Additionally, clinicians must refer those interested in having children to preservation specialists promptly $y^{8,9}$ so they can undertake strategies such as cryopreservation and temporary ovarian suppression with gonadotropin-releasing hormone agonists (GnRHa) during chemotherapy. ${ }^{11}$ However, adherence to these recommendations is often suboptimal in limited-resource countries such as Mexico, presumably because of physicians' lack of knowledge regarding the safety of preservation methods and pregnancy after BC, financial hindrances, scarcity of specialists, and lack of partnerships with reproductive units. ${ }^{12-14}$

Moreover, in resource-constrained settings such as Mexico, cancer control efforts are mainly focused on providing medical treatment, generally bypassing the survivorship aspects of care. ${ }^{15}$ Additionally, preservation strategies are seldom covered by private or governmental healthcare insurances and are unaffordable for most underserved populations. ${ }^{4,16}$

To address these barriers, the "Joven \& Fuerte (Mexican Young \& Strong): Program for Young Women With Breast Cancer" (J\&F) was created in 2014 as the first specialized Latin American program with the goal of comprehensively characterizing a prospective cohort of $\mathrm{YWBC}$, assessing their needs, and offering them supportive care 
services, including enhanced access to FP..$^{15}$ This study describes patients from J\&F who have undergone FP and assesses which sociodemographic and clinicopathologic features were associated with the likelihood of preserving fertility.

\section{Methods}

\section{Description of J\&F}

J\&F is currently active at 3 sites: Instituto Nacional de Cancerologia (INCan) in Mexico City and Hospital San Jose (HSJ) and Hospital Zambrano Hellion (HZH) in Monterrey. The program also receives patients interested in FP referred from Fundacion de Cancer de Mama and Instituto Nacional de Ciencias Medicas y Nutricion Salvador Zubiran in Mexico City. The Mexico City sites and HSJ provide care for patients with public healthcare insurance, whereas HZH receives those with private coverage.

Women aged $\leq 40$ years with recently diagnosed breast cancer are invited to participate in J\&F. The navigator discusses and provides educational material regarding relevant issues for YWBC, identifies patients' needs, and assists in making early referrals to oncofertility, genetic counseling, and psycho-oncology, as needed. Additionally, patients are invited to participate in the prospective cohort for research purposes. A detailed description of the structure and functioning of J\&F has been published previously. ${ }^{15}$

Specifically, to deliver oncofertility services, J\&F has established local alliances with assisted-reproduction units, which provide special-price cryopreservation procedures for underserved patients enrolled in the program. Additionally, for some patients, the coverage of preservation strategies has been possible through governmental support. In this way, J\&F has enhanced access to specialized FP methods that would otherwise be prohibitive due to their high costs. Regarding GnRHa, their use for FP purposes is not covered in some institutions, and they represent an out-of-pocket expense for patients.

\section{Study Design}

This study was developed based on the J\&F prospective cohort. All participants enrolled up to June 2019 were eligible. Data for this analysis were collected from patient-reported information at diagnosis/baseline (age, educational level, employment status, partnership status, number of children, medical affiliation, desire of future biologic children), and provider-recorded data at baseline (clinical stage, breast cancer subtype) and 1-year follow-up (patient use of FP methods, oncologic treatment).

\section{Statistical Analysis}

Patients were categorized into 2 groups: use versus nonuse of a preservation strategy (oocyte/embryo cryopreservation and/or GnRHa for FP). Descriptive statistics were used to analyze the sociodemographic and clinicopathologic characteristics of each group. These variables were then compared between groups using a chi-square test. Simple logistic regression using complete-case analysis was conducted and odds ratios (ORs) and 95\% confidence intervals were calculated to determine the likelihood of preserving fertility. The same analysis was performed to compare patients who underwent FP with those who desired future biologic children at diagnosis but did not preserve fertility. A $P$ value $<.05$ was significant. Statistical analysis was performed using SPSS Statistics, version 25 (IBM Corp).

Additionally, the following time intervals were calculated: diagnosis to neoadjuvant chemotherapy initiation, diagnosis to surgery, and surgery to adjuvant systemic therapy initiation. Medians were compared between patients who underwent cryopreservation and those who did not to determine whether any delays occurred in the start of oncologic treatment as a result of FP.

\section{Results}

This study included 447 patients, 265 (59\%) from the Mexico City sites (mainly from INCan), 140 (31\%) from HSJ, and 42 (9\%) from HZH. Median age at diagnosis was 36 years. Most participants had at least a high school education (57\%), were unemployed/housewives (65\%), were publicly insured $(90 \%)$, and were married/in domestic partnerships (64\%). At diagnosis, $21 \%$ were childless and $31 \%$ desired (more) biologic children. Table 1 shows participants' remaining characteristics.

Of the total patients, 53 (12\%) underwent FP, representing $38 \%$ of the 140 who desired future biologic children at the time of the baseline survey. Of these 53 patients, 28 (53\%) were enrolled at the Mexico City sites, $12(23 \%)$ at HSJ, and $13(25 \%)$ at HZH. Oocyte/embryo cryopreservation was the most frequently used method (59\%), followed by temporary ovarian suppression with GnRHa during chemotherapy (26\%), and oocyte/embryo cryopreservation along with GnRHa (15\%; Figure 1).

At the Mexico City sites, 25 (89\%) patients underwent oocyte/embryo cryopreservation (22 provided through a dedicated governmental fund, at no cost to patients) and $6(21 \%)$ received GnRHa (all covered by the public healthcare system). At HSJ, 7 (58\%) cryopreserved oocytes/embryos (5 offered with a special discount) and 6 (50\%) used GnRHa (all covered out-of-pocket by patients). At HZH, 7 (54\%) underwent cryopreservation (all paid for by patients) and $10(77 \%)$ used GnRHa (covered by private insurances).

Patient characteristics associated with a significantly higher likelihood of preserving fertility were age $\leq 36$ years (OR, 3.0; 95\% CI, 1.5-6.1), having at least high school education (OR, 15.7; 95\% CI, 4.7-65.6), being 


\begin{tabular}{|c|c|}
\hline Characteristic & $n(\%)$ \\
\hline Total, $\mathrm{n}$ & 447 \\
\hline Age, median (range), y & $36(32-38)$ \\
\hline \multicolumn{2}{|l|}{ Educational level } \\
\hline No schooling & $2(0.4)$ \\
\hline Elementary school & $40(8.9)$ \\
\hline Middle school & 107 (23.9) \\
\hline High school & $69(15.4)$ \\
\hline Technical career & $39(8.7)$ \\
\hline Undergraduate or college & $128(28.6)$ \\
\hline Postgraduate & $20(4.5)$ \\
\hline Missing & $42(9.4)$ \\
\hline \multicolumn{2}{|l|}{ Employment status } \\
\hline Employed & $140(31.3)$ \\
\hline Unemployed/Housewife & $292(65.3)$ \\
\hline Student & $15(3.4)$ \\
\hline Disabled & $19(4.3)$ \\
\hline Missing & $11(2.5)$ \\
\hline \multicolumn{2}{|l|}{ Insurance status } \\
\hline Public insurance & $402(89.9)$ \\
\hline Private insurance & $42(9.4)$ \\
\hline Missing & $3(0.7)$ \\
\hline \multicolumn{2}{|l|}{ Partnership status } \\
\hline Unpartnered & $132(29.5)$ \\
\hline Partnered & $287(64.2)$ \\
\hline Missing & $28(6.3)$ \\
\hline \multicolumn{2}{|l|}{ Number of children } \\
\hline None & $92(20.6)$ \\
\hline 1 & $87(19.5)$ \\
\hline$\geq 2$ & $240(53.7)$ \\
\hline Missing & $28(6.3)$ \\
\hline
\end{tabular}

(continued)

employed (OR, 3.9; 95\% CI, 2.1-7.2), having private insurance (OR, 4.3; 95\% CI, 2.0-9.1), and having one or no children (OR, 26.8; 95\% CI, 6.0-119.4, and OR, 63.5; 95\% CI, 14.8-272.3, respectively). When considering only patients who desired future biologic children, the same variables were significantly associated with a higher likelihood of pursuing FP, except for age $\leq 36$ years (Table 2).

Of the 87 patients who wanted (more) biologic children but did not preserve fertility, $38(44 \%)$ reported the following reasons for not doing so: needing to start cancer treatment immediately $(n=19 ; 22 \%)$, personal reasons $(n=11 ; 13 \%)$, and financial issues $(n=10 ; 11 \%)$. Among patients from Mexico City, financial issues

\begin{tabular}{|c|c|}
\hline Characteristic & n (\%) \\
\hline \multicolumn{2}{|c|}{ Desire to have (more) biologic children at diagnosis } \\
\hline Yes & $140(31.3)$ \\
\hline No & 307 (68.7) \\
\hline \multicolumn{2}{|l|}{ Clinical stage } \\
\hline 0 & $5(1.1)$ \\
\hline 1 & $54(12.1)$ \\
\hline II & $199(44.5)$ \\
\hline III & $151(33.8)$ \\
\hline IV & $34(7.6)$ \\
\hline Missing & $4(0.9)$ \\
\hline \multicolumn{2}{|l|}{ Breast cancer subtype } \\
\hline $\mathrm{HR}+/ \mathrm{HER} 2-$ & $234(52.3)$ \\
\hline $\mathrm{HR}+/ \mathrm{HER} 2+$ & 69 (15.4) \\
\hline HR-/HER2+ & $28(6.3)$ \\
\hline Triple-negative & $110(24.6)$ \\
\hline Missing & $7(1.6)$ \\
\hline \multicolumn{2}{|l|}{ Treatment } \\
\hline Chemotherapy & $363(81.2)$ \\
\hline Radiotherapy & $268(59.9)$ \\
\hline Endocrine therapy & $238(53.2)$ \\
\hline Anti-HER2 therapy & $84(18.8)$ \\
\hline
\end{tabular}

Abbreviation: $\mathrm{HR}$, hormone receptor.

were the main reason for $13 \%$, whereas among those from Monterrey, where all preservation strategies were covered by patients, finances represented the main barrier for $47 \%$.

Regarding the systemic treatment prescribed to patients who underwent FP, $83 \%$ received chemotherapy and $55 \%$ endocrine therapy. No significant difference in treatments received was found between patients who preserved fertility and those who did not.

In total, 39 of $53(74 \%)$ patients used cryopreservation as their FP method: 10 (26\%) before receiving neoadjuvant chemotherapy, 10 (26\%) before surgery and adjuvant treatment, and 19 (49\%) after surgery but before adjuvant treatment. Accordingly, the following median intervals were observed: diagnosis to neoadjuvant chemotherapy, 39 days (range, 25-68 days); diagnosis to surgery, 44.5 days (range, 18-80 days); and surgery to adjuvant systemic therapy, 46 days (range, 16-85 days). The corresponding median intervals for patients who did not preserve fertility or who only used GnRHa for FP were 32.5 days (range, 3-89 days), 42 days (range, 3-162 days), and 41 days (range, 4-129 days), respectively. 


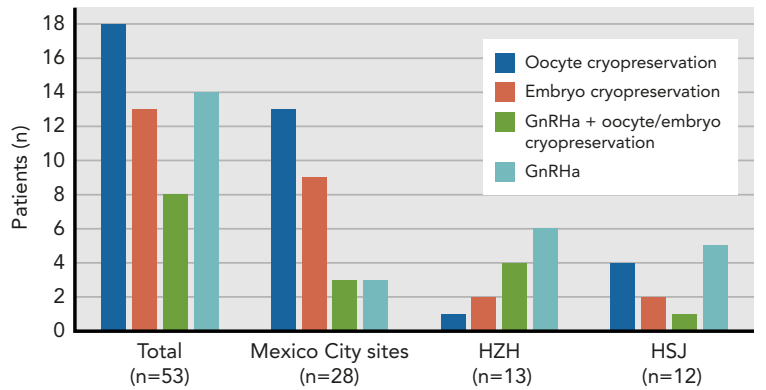

Figure 1. Strategies for fertility preservation in young women with breast cancer from "Joven \& Fuerte."

Abbreviations: GnRHa, gonadotropin-releasing hormone agonists; HSJ, Hospital San Jose; HZH, Hospital Zambrano Hellion.

\section{Discussion}

Although an integral component of cancer care, the implementation of oncofertility practices among YWBC in resource-constrained settings has not become as widespread as in higher income countries. ${ }^{17,18}$ However, this study suggests that specialized supportive care programs that enhance access to preservation procedures for these patients may play a crucial role in reducing that gap.

During a 4 -year period, $12 \%$ of the J\&F patients underwent FP. This proportion is similar to that described in the $\mathrm{HOHO}$ cohort, in which FP measures, including GnRHa use, were practiced among $10 \%$ of patients. ${ }^{19}$ Moreover, this rate represents a significant increase in FP compared with the $<1 \%$ previously reported by our group when J\&F was not yet underway. ${ }^{4}$ Importantly, in a recent cross-sectional study exploring patients' perspectives of J\&F, most participants $(81 \%)$ recalled being informed about the treatment-associated fertility risks. ${ }^{20}$ In contrast, prior to the initiation of J\&F only $31 \%$ of YWBC reported having received this information. ${ }^{4}$ These differences display the positive impact a dedicated program for YWBC can have on promoting a higher number of discussions about reproductive issues and, consequently, more FP referrals, as has also been demonstrated by previous studies in developed countries. ${ }^{21,22}$

More specifically, and possibly of more relevance than the overall implementation rate of FP in this cohort, $38 \%$ of those patients who desired future children were assisted to receive a preservation strategy, a percentage similar to the $31 \%$ reported by a German study of cancer patients in general. ${ }^{23}$ Furthermore, in $70 \%$ of the J\&F cases, the strategy involved oocyte/embryo cryopreservation, the first-line options for FP in patients with cancer. ${ }^{8,11,24}$ This proportion may be explained by the coverage of cryopreservation procedures through a dedicated governmental fund at one of the J\&F sites, as well as the alliances that the program fosters with 4 assistedreproduction centers in Mexico that facilitate referral and provide special discounts for underserved patients who cannot cover these measures' total costs.
It should also be noted that only $41 \%$ of the patients who preserved fertility received GnRHa. This percentage contrasts with current practices in other countries such as Italy, where all YWBC are granted coverage for temporary ovarian suppression with GnRHa during chemotherapy and their use reaches rates of $98 \% .{ }^{25,26} \mathrm{GnRHa}$ are a less expensive, effective, and safe second-line measure for women who have no access, refuse, or have contraindications to cryopreservation procedures. ${ }^{16,27-29}$ Implementing their routine use in the Mexican context, however, is challenging, possibly due to 2 main reasons. First, not all Mexican physicians discuss reproductive issues with YWBC, and a substantial proportion wrongly believe that $\mathrm{GnRHa}$ are detrimental to their prognosis. ${ }^{14}$ Second, GnRHa are seldom covered by public insurances and most patients either lack the resources to afford them or are forced to choose between cryopreserving or receiving GnRHa because of financial constraints. Support from the Mexican public healthcare system through coverage of GnRHa for FP purposes among YWBC, following the examples of countries such as Italy, Sweden, and Israel, ${ }^{25,30,31}$ would aid considerably in addressing this need.

In 2 previous studies from developed countries, the most influential factors in patients' decision to preserve fertility were insurance coverage of the procedures, the ability to pay for them, ${ }^{19}$ and believing prompt cancer treatment initiation was a priority. ${ }^{32}$ Similarly, our patients' main reasons for not undergoing FP even when they desired future biologic children were the perception of needing to start cancer treatment rapidly, personal reasons, and financial issues.

Notably, $27 \%$ of the patients who underwent cryopreservation received neoadjuvant chemotherapy, suggesting that the possibility of delaying this treatment was not a definitive reason to reject FP for a considerable proportion of patients. In this regard, it should be highlighted that no considerable differences were found between patients who cryopreserved and those who did not in terms of the intervals from diagnosis to neoadjuvant chemotherapy initiation (39 vs 32.5 days), from diagnosis to surgery ( 44.5 vs 42 days), or from surgery to adjuvant treatment initiation (46 vs 41 days). According to these findings, undergoing cryopreservation probably does not entail a risk of delaying oncologic treatment. Therefore, it should not represent a decisive factor during patients' and physicians' decision-making when considering FP.

Moreover, the median interval between diagnosis and the start of oncologic treatment was similar to that reported in another study concerning oncofertility and delays in neoadjuvant chemotherapy initiation. ${ }^{33}$ Data are limited regarding the security and outcomes of postponing neoadjuvant chemotherapy to perform FP in patients with breast cancer. ${ }^{33}$ Even though some studies state that this practice is probably safe, ${ }^{30,34,35}$ most patients and 


\begin{tabular}{|c|c|c|c|c|c|c|c|}
\hline Total, $\mathrm{n}$ & 53 & 394 & & & 87 & & \\
\hline Age at diagnosis & & & & .001 & & & .255 \\
\hline$\leq 36 y$ & $39(75)$ & $200(54)$ & $3.0(1.5-6.1)$ & & $60(69)$ & $1.59(0.71-3.58)$ & \\
\hline Educational level & & & & $<.001$ & & & $<.001$ \\
\hline Middle school or less & $2(4)$ & $147(41)$ & Ref & & $27(31)$ & Ref & \\
\hline High school or greater & $45(96)$ & $211(59)$ & $15.7(4.7-65.6)$ & & $59(69)$ & $10.3(2.32-45.5)$ & \\
\hline Disabled & $2(4)$ & $17(5)$ & $0.9(0.2-3.9)^{a}$ & .846 & $5(6)$ & $1.46(0.27-7.83)^{a}$ & .655 \\
\hline Insurance status & & & & $<.001$ & & & .002 \\
\hline Public insurance & $37(76)$ & $355(93)$ & Ref & & $81(94)$ & Ref & \\
\hline Private insurance & $12(24)$ & $27(7)$ & $4.3(2.0-9.1)$ & & $5(6)$ & $5.25(1.72-15.9)$ & \\
\hline Partnership status & & & & .089 & & & .562 \\
\hline Unpartnered & $21(42)$ & $111(30)$ & Ref & & $41(47)$ & Ref & \\
\hline Partnered & $29(58)$ & $258(70)$ & $0.6(0.3-1.1)$ & & $46(53)$ & $1.23(0.61-2.48)$ & \\
\hline Number of children & & & & $<.001$ & & & .001 \\
\hline$\geq 2$ & $2(4)$ & $238(65)$ & Ref & & $25(29)$ & Ref & \\
\hline III & $11(22)$ & $140(36)$ & $0.3(0.03-3.1)$ & & $38(44)$ & $0.28(0.01-5)$ & \\
\hline IV & $1(2)$ & $33(8)$ & $0.1(0.006-2.3)$ & & $3(3)$ & $0.3(0.009-11.9)$ & \\
\hline Breast cancer subtype & & & & .513 & & & .912 \\
\hline $\mathrm{HR}+/ \mathrm{HER} 2-$ & $27(54)$ & $207(53)$ & Ref & & $47(55)$ & Ref & \\
\hline $\mathrm{HR}+/ \mathrm{HER}+$ & $11(22)$ & $58(15)$ & $1.3(0.6-2.9)$ & & $15(17)$ & $1.16(0.45-2.94)$ & \\
\hline HR-/HER2+ & $2(4)$ & $26(7)$ & $0.6(0.1-2.6)$ & & $5(6)$ & $0.69(0.12-3.83)$ & \\
\hline Triple-negative & $10(20)$ & $100(25)$ & $0.7(0.3-1.5)$ & & $19(22)$ & $0.82(0.32-2.07)$ & \\
\hline
\end{tabular}

Abbreviations: FP, fertility preservation; $\mathrm{HR}$, hormone receptor; $\mathrm{OR}$, odds ratio.

aParticipants could select more than one answer for this question. Therefore, reference values correspond to those who were not employed, not unemployed, not students, and not disabled, respectively.

providers are reluctant to delay chemotherapy. ${ }^{36}$ This is particularly relevant in developing countries, in which YWBC frequently present with locally advanced breast cancer that requires neoadjuvant chemotherapy, ${ }^{37,38}$ which might influence decision-making processes regarding FP. Notably, in a context unrelated to FP, 2 studies reported that a delay in neoadjuvant chemotherapy initiation of $\geq 56$ or $\geq 61$ days was associated with an increased risk of death. ${ }^{39,40}$ Considering this, our patients are probably in a safe time frame given that their median time to neoadjuvant chemotherapy was 39 days, with only 1 patient presenting an interval of $\geq 56$ days.

Of note, in relation to financial concerns, more than half of these patients' preservation methods were provided 
at no cost or with special discounts. Additionally, in Monterrey, where patients had to pay for all strategies, a higher proportion listed financial reasons as their main impediment to undergoing FP. Therefore, the extent to which finances represent a barrier for Mexican patients is probably greater than what is reported by this cohort.

According to our comparative analysis, younger age, higher educational level, and being employed were each associated with a higher likelihood of undergoing FP. These characteristics have been previously associated with more fertility concerns and may play a role in the decision-making of YWBC who are interested in preserving fertility. ${ }^{7}$ Moreover, these features could be interrelated, as better-educated women tend to pursue career development and delay childbearing. ${ }^{41-43}$ Hence, YWBC with these characteristics might not have children yet and may be more interested in pursuing FP.

Regarding parity, patients who had one or, especially, no children, were the most likely to preserve fertility. Other studies have also reported that nulliparous YWBC were more likely to have fertility concerns, to desire future children, and to consider seeking FP. ${ }^{7,44}$ In contrast, others have documented that parity did not affect fertility referrals and was not a predictive factor for undergoing FP. ${ }^{45,46}$ Still, considering that being childless at diagnosis was the most prominent predictor of pursuing FP in the present study, physicians could give special emphasis to the treatment-related risk of infertility and the available preservation options among nulliparous patients.

Another finding from this study, although not statistically significant, was a trend toward lower odds of preserving fertility as patients' clinical stage increased. This pattern was also observed in another multicenter analysis that found that patients with lower-stage breast cancer pursued FP more often. ${ }^{47}$ A possible explanation may be that physicians favor discussing fertility issues and making oncofertility referrals when patients have early-stage breast cancer. ${ }^{45}$ Remarkably, despite this trend, one J\&F patient with metastases underwent cryopreservation, which highlights the importance of addressing patients' reproductive concerns on a case-by-case basis and offering FP methods to all young patients, irrespectively of their disease stage. ${ }^{48}$ Nonetheless, FP in patients with poor prognosis raises significant ethical dilemmas, such as the morality of denying FP when life expectancy is limited, the possibility of instilling false hopes of survival by offering FP options, and the matter of coverage for these strategies. ${ }^{49}$ Importantly, given that a reduced lifespan is usually not a sufficient reason to withhold reproductive assistance, ${ }^{50,51}$ physicians' main role should be to help patients make an informed, conscious choice by ensuring their appropriate understanding of prognosis, values and priorities, cost of procedures, and future arrangements for the child's welfare, always respecting patient autonomy. ${ }^{52-54}$
As previously emphasized by others, fertility concerns are not limited to patients who possess specific characteristics. ${ }^{4,55}$ Patients' features must not be regarded as determinants when physicians consider addressing reproductive issues and making referrals to oncofertility units. On the contrary, all YWBC must be informed about these topics and offered FP options, regardless of physicians' preconceptions.

This study has important strengths and limitations. The main positive aspects include its prospective and longitudinal design and the recruitment of patients from 3 of the country's main breast cancer referral centers. Additionally, it provides evidence that asserts the benefits and need for dedicated programs for YWBC. However, given that the studied population belongs to a special supportive care program for this young group, their results could differ substantially from patients who are not members of similar organizations. Last, other unmeasured variables possibly exist between patients who preserved fertility and those who did not, including rates of referral to oncofertility counseling, time between referral and appointments, and the impact of such consultations on FP decision-making, which could influence patients' likelihood of undergoing FP.

\section{Conclusions}

This study represents the largest FP report from a prospective cohort of YWBC in a resource-constrained country. Among these patients, younger age, higher educational level, being employed, being privately insured, and having 1 or no children were associated with a higher likelihood of preserving fertility. These characteristics should not be regarded as determinant identifiers of patients who will pursue FP, but they may aid oncologists in recognizing women with whom fertility issues and preservation strategies should be particularly underscored.

Furthermore, these findings suggest that median intervals until oncologic treatment initiation do not differ between women who cryopreserve and those who do not. With this information, physicians might reassure patients that the time needed for FP should not delay the start of their oncologic treatment or negatively impact their outcomes. Hence, this concern should not be a decisive factor for patients or clinicians when considering FP. Nonetheless, this observation should be explored in future studies and efforts should be made to decrease these intervals further.

Finally, this study also highlights the transcendence that a directed, supportive care program for YWBC can have on enhancing access to specialized oncofertility services, particularly in limited-resource settings. Certainly, increased physician awareness and advocacy for FP in YWBC are needed for these efforts to be successful and widely applied in the long term. Moreover, sustained and extended governmental support, like that offered in other countries, is warranted for the routine coverage of 
FP for YWBC. These practices will ultimately translate into delivering integral care to this group.

\section{Acknowledgments}

Programa para contribuir a mejorar el tratamiento integral con equipo multidisciplinario a las pacientes con diagnóstico de cáncer de mama, Centro Medico ABC, Clinica de reproduccion asistida HISPAREP - Hospital Español, Clinica de fertilidad/ reproduccion asistida IVINSEMER, Centro de Reproduccion Asistida CREASIS, Instituto para el estudio de la concepcion humana - Hospital Ginequito.

Submitted May 5, 2020; revision received October 31, 2020; accepted for publication November 30, 2020

Published online June 21, 2021.
Author contributions: Study concept and design: Villarreal-Garza, Plata de la Mora, Miaja-Avila, García Garza, Mohar, Bargallo-Rocha. Material preparation: Mesa-Chavez, Garcia-Garcia, Fonseca, de la Rosa-Pacheco, Cruz-Ramos. Data acquisition: Mesa-Chavez, Garcia-Garcia, Fonseca, de la Rosa-Pacheco, Cruz-Ramos. Data analysis and interpretation: Mesa-Chavez, Garcia-Garcia, Fonseca, de la Rosa-Pacheco, Cruz-Ramos. Manuscript preparation: Villarreal-Garza, Mesa-Chavez, Garcia-Garcia. Critical revision: All authors. Final approval of manuscript: All authors.

Disclosures: The authors have disclosed that they have not received any financial consideration from any person or organization to support the preparation, analysis, results, or discussion of this article.

Correspondence: Enrique Bargallo-Rocha, MD, Departamento de Tumores Mamarios e Investigacion, Instituto Nacional de Cancerologia, San Fernando 22, Belisario Dominguez Secc 16, Tlalpan, Ciudad de Mexico, Mexico, 14080. Email: enrique.bargallo@gmail.com; and

Cynthia Villarreal-Garza, MD, DSc, Centro de Cancer de Mama-TecSalud Tecnologico de Monterrey, Batallon de San Patricio 112, 14th Floor, Real de San Agustin, San Pedro Garza Garcia, Nuevo Leon, Mexico, 66278. Email: cynthia.villarreal@tecsalud.mx; cynthiavg@gmail.com

\section{References}

1. Bray F, Ferlay J, Soerjomataram I, et al. Global cancer statistics 2018: GLOBOCAN estimates of incidence and mortality worldwide for 36 cancers in 185 countries. CA Cancer J Clin 2018;68:394-424.

2. Lawrenz B, Jauckus J, Kupka MS, et al. Fertility preservation in $>1000$ patients: patient's characteristics, spectrum, efficacy and risks of applied preservation techniques. Arch Gynecol Obst 2011;283:651-656.

3. Villarreal-Garza C, Aguila C, Magallanes-Hoyos MC, et al. Breast cancer in young women in Latin America: an unmet, growing burden. Oncologist 2013:18:1298-1306.

4. Villarreal-Garza C, Martinez-Cannon BA, Platas A, et al. Fertility concerns among breast cancer patients in Mexico. Breast 2017;33:71-75.

5. Letourneau JM, Ebbel EE, Katz PP, et al. Pretreatment fertility counseling and fertility preservation improve quality of life in reproductive age women with cancer. Cancer 2012;118:1710-1717.

6. Rosen A, Rodriguez-Wallberg KA, Rosenzweig L. Psychosocial distress in young cancer survivors. Semin Oncol Nurs 2009;25:268-277.

7. Partridge $\mathrm{AH}$, Gelber S, Peppercorn J, et al. Web-based survey of fertility issues in young women with breast cancer. J Clin Oncol 2004;22:4174-4183

8. Loren AW, Mangu PB, Beck LN, et al. Fertility preservation for patients with cancer: American Society of Clinical Oncology clinical practice guideline update. J Clin Oncol 2013;31:2500-2510.

9. Peccatori FA, Azim HA Jr, Orecchia R, et al. Cancer, pregnancy and fertility: ESMO Clinical Practice Guidelines for diagnosis, treatment and follow-up. Ann Oncol 2013;24(Suppl 6):vi160-170.

10. Consenso Mexicano sobre diagnóstico y tratamiento del cáncer mamario. Octava revisión. Accessed February 23, 2021. Available at: http:// consensocancermamario.com/documentos/FOLLETO_CONSENSO_DE CANCER_DE_MAMA_8aRev2019a.PDF

11. Lambertini M, Del Mastro L, Pescio MC, et al. Cancer and fertility preservation: international recommendations from an expert meeting. BMC Med 2016;14:1

12. Goossens J, Delbaere I, Van Lancker A, et al. Cancer patients' and professional caregivers' needs, preferences and factors associated with receiving and providing fertility-related information: a mixed-methods systematic review. Int J Nurs Stud 2014;51:300-319.

13. Rashedi AS, de Roo SF, Ataman LM, et al. Survey of fertility preservation options available to patients with cancer around the globe. J Glob Oncol 2018:4:1-16.

14. Villarreal-Garza C, Barragan-Carrillo R, Bargallo-Rocha JE, et al. Physician knowledge and attitudes toward fertility preservation in Mexican young breast cancer patients [abstract]. Cancer Res 2018;78(Suppl):Abstract P6-11-09.

15. Villarreal-Garza C, Castro-Sánchez A, Platas A, et al. "Joven \& Fuerte": program for young women with breast cancer in Mexico-initial results. Rev Investig Clin 2017;69:223-228.

16. Lambertini M, Goldrat O, Barragan-Carrillo R, et al. Viable options for fertility preservation in breast cancer patients: a focus on Latin America. Rev Investig Clin 2017;69:103-113.
17. Anazodo A, Ataman-Millhouse L, Jayasinghe $Y$, et al. Oncofertility: an emerging discipline rather than a special consideration. Pediatr Blood Cancer 2018;65:e27297.

18. Anazodo A, Laws $P$, Logan $S$, et al. The development of an international oncofertility competency framework: a model to increase oncofertility implementation. Oncologist 2019;24:e1450-1459.

19. Ruddy KJ, Gelber SI, Tamimi RM, et al. Prospective study of fertility concerns and preservation strategies in young women with breast cancer. J Clin Oncol 2014;32:1151-1156.

20. Villarreal-Garza C, Platas A, Miaja M, et al. Patients' satisfaction with a supportive care program for young breast cancer patients in Mexico: Joven \& Fuerte supports patients' needs and eases their illness process. Support Care Cancer 2020;28:4943-4951.

21. Vu JV, Llarena NC, Estevez SL, et al. Oncofertility program implementation increases access to fertility preservation options and assisted reproductive procedures for breast cancer patients. J Surg Oncol 2017;115:116-121.

22. Srikanthan A, Amir E, Warner E. Does a dedicated program for young breast cancer patients affect the likelihood of fertility preservation discussion and referral? Breast 2016;27:22-26.

23. Geue K, Richter D, Schmidt R, et al. The desire for children and fertility issues among young German cancer survivors. J Adolesc Heal 2014:54:527-535.

24. Ronn R, Holzer H. Breast cancer and fertility: an update. Curr Opin Support Palliat Care 2015;9:285-293.

25. Lambertini M, Cinquini M, Moschetti I, et al. Temporary ovarian suppression during chemotherapy to preserve ovarian function and fertility in breast cancer patients: a GRADE approach for evidence evaluation and recommendations by the Italian Association of Medical Oncology. Eur J Cancer 2017:71:25-33

26. Lambertini M, Fontana V, Massarotti $C$, et al. Prospective study to optimize care and improve knowledge on ovarian function and/or fertility preservation in young breast cancer patients: results of the pilot phase of the PREgnancy and FERtility (PREFER) study. Breast 2018:41:51-56.

27. Moore HCF, Unger JM, Phillips KA, et al. Final analysis of the prevention of early menopause study (POEMS)/SWOG intergroup S0230. J Natl Cancer Inst 2019;111:210-213.

28. Lambertini M, Boni L, Michelotti A, et al. Ovarian suppression with triptorelin during adjuvant breast cancer chemotherapy and long-term ovarian function, pregnancies, and disease-free survival a randomized clinical trial. JAMA 2015;314:2632-2640.

29. Lambertini M, Richard F, Nguyen B, et al. Ovarian function and fertility preservation in breast cancer: should gonadotropin-releasing hormone agonist be administered to all premenopausal patients receiving chemotherapy? Clin Med Insights Reprod Heal 2019;13:1179558119828393.

30. Rodriguez-Wallberg KA, Eloranta S, Krawiec K, et al. Safety of fertility preservation in breast cancer patients in a register-based matched cohort study. Breast Cancer Res Treat 2018;167:761-769.

31. Birenbaum-Carmeli D. Thirty-five years of assisted reproductive technologies in Israel. Reprod Biomed Soc Online 2016;2:16-23. 
32. Pursche T, Bauer J, Hammersen F, et al. Early-onset breast cancer: effect of diagnosis and therapy on fertility concerns, endocrine system, and sexuality of young mothers in Germany. Breast Care (Basel) 2019;14:23-29.

33. Letourneau JM, Sinha N, Wald K, et al. Random start ovarian stimulation for fertility preservation appears unlikely to delay initiation of neoadjuvant chemotherapy for breast cancer. Hum Reprod 2017;32:2123-2129.

34. Ter Welle-Butalid MEE, Vriens IJHI, Derhaag JGJ, et al. Counseling young women with early breast cancer on fertility preservation. J Assist Reprod Genet 2019;36:2593-2604.

35. Lambertini M, Fontanella C. How reliable are the available safety data on hormonal stimulation for fertility preservation in young women with newly diagnosed early breast cancer? Breast Cancer Res Treat 2018;168:773-774.

36. Lambertini M, Di Maio M, Pagani O, et al. The BCY3/BCC 2017 survey on physicians' knowledge, attitudes and practice towards fertility and pregnancy-related issues in young breast cancer patients. Breast 2018;42:41-49.

37. Villarreal-Garza C, Mohar A, Bargallo-Rocha JE, et al. Molecular subtypes and prognosis in young Mexican women with breast cancer. Clin Breast Cancer 2017;17:e95-e102.

38. Villarreal-Garza C, Lopez-Martinez EA, Muñoz-Lozano JF, et al. Locally advanced breast cancer in young women in Latin America. Ecancermedicalscience 2019;13:894.

39. Sanford RA, Lei X, Giordano SH, et al. Impact of delayed neoadjuvant systemic chemotherapy on survival outcomes in breast cancer patients [abstract]. J Clin Oncol 2016;34(Suppl):Abstract 1038.

40. Chavez Mac Gregor M, Lei X, Gagliato D, et al. Impact of delayed neoadjuvant systemic chemotherapy on survival among breast cancer patients. Ann Oncol 2019;30:v71

41. Amuedo-Dorantes C, Kimmel J. The motherhood wage gap for women in the United States: the importance of college and fertility delay. Rev Econ Househ 2005;3:17-48.

42. Rendall M, Couet C, Lappegard T, et al. First births by age and education in Britain, France and Norway. Popul Trends 2005;121:27-34.
43. Martin SP. Diverging fertility among U.S. women who delay childbearing past age 30. Demography 2000;37:523-533.

44. Goldfarb SB, Kamer SA, Oppong BA, et al. Fertility preservation for the young breast cancer patient. Ann Surg Oncol 2016;23:1530-1536.

45. Lee S, Heytens E, Moy F, et al. Determinants of access to fertility preservation in women with breast cancer. Fertil Steril 2011;95:1932-1936.

46. Snyder KA, Tate AL. What to do now? How women with breast cancer make fertility preservation decisions. J Fam Plan Reprod Heal Care 2013;39:172-178.

47. Kim J, Oktay K, Gracia C, et al. Which patients pursue fertility preservation treatments? A multicenter analysis of the predictors of fertility preservation in women with breast cancer. Fertil Steril 2012;97:671-676.

48. Paluch-Shimon S, Cardoso F, Partridge AH, et al. ESO-ESMO 4th international consensus guidelines for breast cancer in young women (BCY4). Ann Oncol 2020;31:P674-691.

49. Linkeviciute A, Boniolo G, Peccatori FA. Setting up an ethical oncofertility practice in developing countries. Bangladesh J Bioeth 2014; 5:6-17.

50. Robertson JA. Cancer and fertility: ethical and legal challenges. J Natl Cancer Inst Monogr 2005;2005:104-106.

51. The Ethics Committee of the American Society for Reproductive Medicine. Fertility preservation and reproduction in patients facing gonadotoxic therapies: a committee opinion. Fertil Steril 2013;100:1224-1231.

52. Walsh SK, Ginsburg ES, Lehmann LS, et al. Oncofertility: fertile ground for conflict between patient autonomy and medical values. Oncologist 2017;22:860-863.

53. Gracia CR, Gracia JJE, Chen S. Ethical dilemmas in oncofertility: an exploration of three clinical scenarios. Cancer Treat Res 2010;156:195-208.

54. Meyer F, Farrell E. Ethical dilemmas in palliative care: a case study of fertility preservation in the context of metastatic cancer. J Palliat Med 2015;18:661.

55. Yee S, Abrol K, McDonald M, et al. Addressing oncofertility needs: views of female cancer patients in fertility preservation. J Psychosoc Oncol 2012;30:331-346. 